\title{
Ultrastructure and Division Behaviour of Dinoflagellate Chromosomes II. Quantitative three-dimensional organization and karyotype of Prorocentrum triestinum ${ }^{1}$
}

\author{
E. Costas and V. J. Goyanes \\ Section of Genetics, Children and Maternal Center, "Juàn Canalejo" Hospital, \\ Las Jubias s/n, La Coruña E-15006, Spain, and \\ Genetics and Pathology Dpt., Regional Oncology Center \\ C/Monserrat, La Coruña E-15006, Spain
}

Accepted August 8, 1988

Dinoflagellate microalgae present a variety of nuclear features which in evolutionary terms classify them apart from the actual eukaryotes (Herzog et al. 1984). Their chromosomes remain permanently condensed along the cell cycle (Dodge 1963), and lack histones and nucleosomes (Loeblich 1976, Rizzo 1981, Herzog and Soyer 1981). Also, DNA replication and chromosome division develop as coupled events (Costas and Goyanes 1987).

Under the electron microscope thin sectioned chromosomes show a periodic and fibrilar arrangement constituted by stacked series of parallel nested arches (Livolant and Bouligand 1978). When dinoflagellate chromosomes were spread to analyze their whole structure, they presented a helical arrangement (Oakley and Dodge 1979, Livolant and Bouligand 1980, Herzog and Soyer 1983, Soyer and Herzog 1985). These ultrastructural facts led to interpret the chromosome organization as a liquid cholesteric crystal (Livolant and Bouligand 1978, 1980), a double helical toroidal bundle (Oackley and Dodge 1979), or a DNA hierarchy of six organization levels (Herzog et al. 1984). Analyses of dinoflagellates chromosomal dimensions only were scarcely reported either in thin sectioned material or spread chromosomes (Livolant and Bouligand 1980).

In a previous report we presented the qualitative features of the three-dimensional organization of Prorocentrum triestinum and Proracentrum micans chromosomes (Costas and Goyanes 1987). Here we morphometrically evaluate the whole-mounted chromosomes of a dinoflagellate, as well as its karyotype for the first time.

Material and methods

\section{Cultures}

Prorocentrum triestinum (Schiller) strains, isolated from Galician shores (NW Spain), were grown under $25 \mu$ Ein $\mathrm{m}^{-2} \mathrm{~S}^{-1}$ continuous light at $20^{\circ} \mathrm{C}$ in $\mathrm{f} / 2$ medium. Free running and exponentially growing cells were centrifuged $10 \mathrm{~min}$ at $1,200 \mathrm{rpm}$ and processed either by whole-mounting or thin sectioning.

\section{Electron microscopy}

A) Isolation and whole-mounting. They were performed as previously described (Costas and Goyanes, 1987). In short:

Non-disrupted pellets of $2.10^{5}$ microalgae were covered with $2 \mathrm{ml}$ of isolation buffer $\left(1 \%\right.$ citric acid, $1 \%$ triton $\mathrm{X}-100$ and $6 \mathrm{mM} \mathrm{MgCl} 2$ ) for $30 \mathrm{~min}$ at $20^{\circ} \mathrm{C}$. Dinoflagellates were suspended in the isolation buffer and chromosomes isolated by 10 passes of a loose pestle

\footnotetext{
${ }^{1}$ Supported by CAICYT Grant 2409/83.
} 
in a dounce homogeneizer. The chromosomes were centrifuged for $5 \mathrm{~min}$ at $2000 \mathrm{rpm}$ through $2 \mathrm{ml}$ isolation buffer onto formwar, coated electron microscope grids. Afterwards, the grids were immersed into consecutive $10 \mathrm{~min}$ baths of $50 \%, 75 \%$ and $100 \%$ ethanol and two $5 \mathrm{~min}$ baths of amyl acetate.

B) Embedding and thin sectioning. They were performed as previously described (Costas and Goyanes, 1987). More in detail:

Pellets of $10^{5}$ cells were fixed in $2 \%$ glutaraldehide and $1 \%$ osmium tetroxid in sodium cacodilate sacharose buffer. Embedding was performed in Epon and seventy nm. thick sections were stained with uranyle acetate followed by lead citrate. To check the effect of the isolation buffer on the chromosome structure, undisrupted pellets were covered for 30 min by the isolation buffer and embedded in Epon after progressive alcoholic baths. Then thin cut and stained as described.

\section{Morphometry and chromosome karyotyping}

Morphometry was a previously described (Costas and Goyanes, 1987). In short:

Electron microscope examination and photographic recording were performed in a Zeiss 109 turbo E.M. operated at $85 \mathrm{kvol}$., employing latex bead of $109 \mathrm{~nm}$ diameter as size markers.

We measured the axial length, chromosome diameter and coils number of 164 wholemounted chromosomes. From these data the longest, the shortest as well as the average $P$. triestinum chromosome were morphometrically characterized.

In an attempt to establish the $P$. triestinum karyotype we classify all the 64 chromosomes by their morphometric criteria of axial length, coils number, $\lambda$ and CF coefficients of form. These two later dimensionless coefficients were determined from the estimated perimeter and area profiles of the chromosomes employing curvimeters and point-counting procedures respectively (Baak and Oort 1983). The coefficient of form CF (Renau-Piqueras and Cervera 1983, Renau-Piqueras et al. 1983) was determined according to the expression:

$$
\mathrm{CF}=4 \pi \mathrm{a} / \mathrm{p}^{2}
$$

where $\mathrm{a}$ is the chromosome profile area and $\mathrm{p}$ the chromosome perimeter. The coefficient of from $\lambda$ was determined as the ratio of chromosome length to chromosome diameter (or mean axial ratio of the chromosome profile) (Weibel and Bolender 1973).

\section{Results and discussion}

\section{Quantitative three-dimensional organization}

Although the fibrilar organization of dinoflagellate chromosomes has been extensively studied on thin sectioned material (Livolant and Bouligand 1978, Oackley and Dodge 1979, Spector et al. 1981), the higher levels of their chromosome architecture were preferentially analyzed on spread or isolated chromosomes. Thus, Haapala and Soyer (1973) and Livolant and Bouligand (1979) described dinoflagellate chromosomes as a double helical bundle after water spreading or squash, respectively. Oackley and Dodge (1979) on spread chromosomes reported them as a toroidal bundle coiling plectonemically into a tigh double helix.

In this respect we previously proposed a procedure to isolate and fix dinoflagellate chromosomes in suspension, then getting three-dimensionally well preserved samples (Costas and Goyanes 1987). All the chromosomes showed an identical double helical left-handed organization constituted by two narrow wings tightly coiling and ending by differentiated telomeres (Costas and Goyanes 1987). When carefully examined two kinds of coils were identified: a) An upper region constituted by two wings coiling together for the first two turns, and b) A lower region where wings progressively separate, while their tilt angle increases with respect 


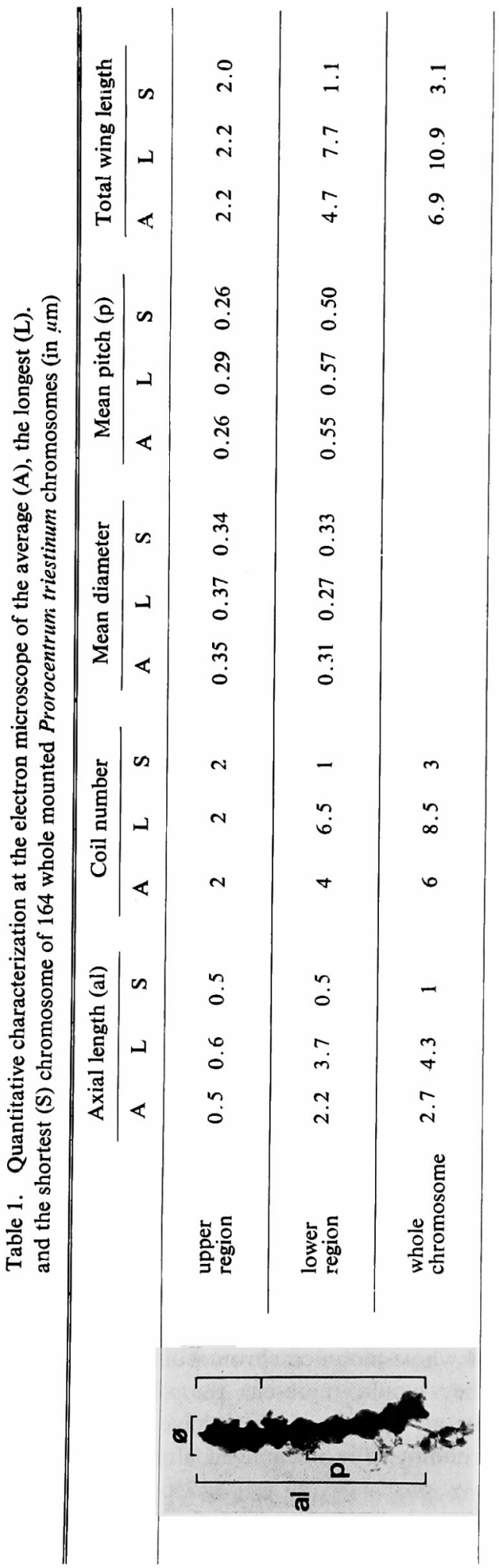


to the chromosome axis (Costas and Goyanes 1987).

In an attempt to morphometrically characterize these chromosomes, we measured the axial chromosome length, diameter, coil number, pitch, tilt angle and total length of the wings in $164 \mathrm{P}$. triestinum chromosomes. Table 1 compares the dimensions of the longest, the shortest and the average chromosome. Although the respective upper regions show to be similar, differences between chromosomes were found in the lengths, tilt angles and coil number of their lower regions. When their general dimensions are compared, the longest chromosome shows to be 4 times longer and to have three times more turns than the shortest one. The tilt angle which presents a value of around $13^{\circ}$ for the first two coils, progressively increased $4^{\circ}$ each of successive coils till the lower telomere. As a consecuence of this the chromosome diameter decreases downward. Our stimated chromosome lengths and diameters are similar to reported by Oakley and Dodge (1979) in Glenodinium hallii and Amphidinium carterae employing a careful fixation procedure and serial sectioning. Also Blank (1987) in a three-dimensional reconstruction of a cell of Synbiodinium sp. found coincidental chromosome magnitudes.

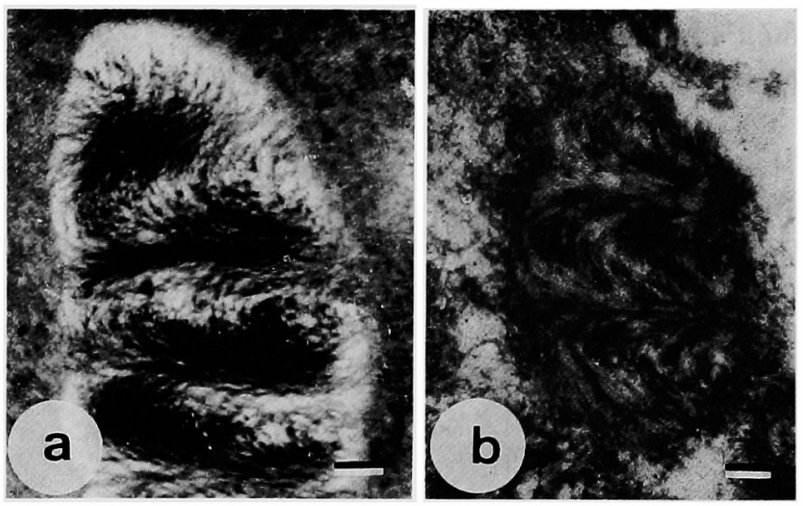

Fig. 1. To evaluate the possible effects of the isolation buffer on the chromsome organization, thin sectioned chromosomes fixed by glutaraldehide-osmium procedure (a) and treated by the isolation buffer (b) are compared. Both samples show an identical pattern of stacked series of arches, suggesting no artefactual consequences of the whole mounting procedure. The isolation buffer paratially removes the electrondense nuclear matrix. Bar $0.1 \mu \mathrm{m}$.

To evaluate the possible effects of the isolation buffer on the chromosome structure we embedded and thin sectioned $P$. triestinum cells only previously treated by the buffer. When chromosomes fixed by $2 \%$ glutaraldehyde and $1 \%$ osmium tetroxide were compared with the buffer treated samples, a similar pattern of stacked series of arches was observed (Fig. 1) then suggesting no main artefactual consequences of our whole mounting procedure.

Some consequences on the chromosome organization can also be drawn from culture conditions. All of the 164 whole-mounted chromosomes herein analyzed were obtained from free-running cultures. They would represent the all possible chromosome morphologies along the cell cycle. Since no changes in them have been detected, it can be inferred that chromosomes remain morphologically unchanged along the cell kinetics except for their replication-division period.

A word also on the physical properties of dinoflagellate chromosomes as it can be deduced from the striking fact that all the whole-mount chromosomes appeared straight rod-shape on the grid, while they bend when remaining within the nucleus. Provided that in the whole- 
mount procedure the chromosome is released from the nuclear matrix and nucleoplasm, then it can be deduced that these would influence the chromosome morphology when chromosomes are still located within the nucleus. Moreover, in the absence of the dense nuclear environment and nucleolar envelope that embrace and bend the chromosome, it seems to behave as a spring where its intrinsecal physical tensions equilibrate, thus straightening the chromosome.

The spatial organization of dinoflagellate chromosomes closely resembles the Whitwarth's screw of the 19th Century; in this respect we proposed to denominate them as "screw chromosomes" (Costas and Goyanes 1987). From a topological point of view the upper region would assimilate to an "ear-ring" twisted, being the lower region organized as a torsion.

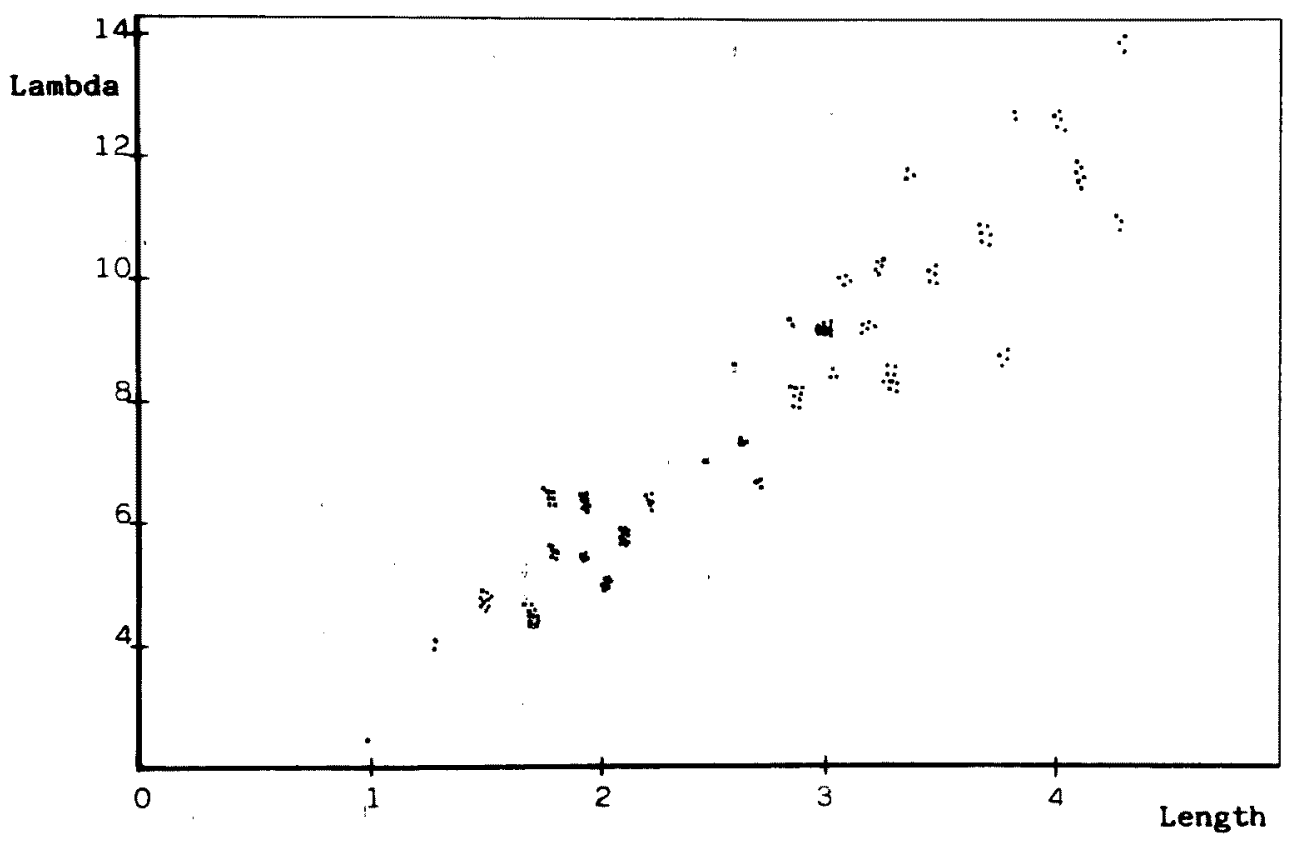

Fig. 2. $164 P$. triestinum whole-mounted chromosomes ordered by either their respectives axial lengths and lambda coefficients of form. Scatter shows 32 groups which would correspond to chromosomes 1 to 32 from Table 2 and Fig. 3.

\section{The karyotype}

The lack of morphological qualitative differences among chromosomes, the absence of response to bands-induction (Herzog et al. 1984), their small dimensions, as well as spreading difficulties have prevented until now the karyotyping of the dinophyceae phylum. Thus a morphometric approach employing EM could be one of the valid tentatives to establish it. In fact Bahr and Goulomb (1971) karyotyped human chromosomes from its total dry mass and the ratio of long to short arms mass determined by electron microscopy. Lampert et al. (1969) quantitatively characterized by electron microscopy a marker chromosome of a Burkitt's lymphoma in terms of dry mass and DNA packing ratio. Du Praw (1970) described the human chromosomes in terms of mathematical equations.

Previous stereological data pointed out that $P$. triestinum complement consists on 32 chromosomes. (Costas and Goyanes 1987). Although similar in their general structure, when our 164 chromosomes, were ordered by their axial length and lambda coefficient of form (Weibel and Bolender 1973), 32 groups appeared as expected (Fig. 2). 
In Table 2 these 32 groups are ordered by the mean values of lenght and lambda of the chromosomes which compose each group. Thus, the groups are numbered from 1 to 32, which would correspond to each one of the chromosomes of the $P$. triestinum karyotype. It is also noticed that the number of coils is directly related to the chromosome lenght (coefficient of correlation $=0.938$ ). In Fig. 3 the quantitative $P$. triestinum karyotype is presented based on the values given in Table 2. Related to the existence of sex chromosomes in the $P$. triestinum karyotype we have failed to find chromosomes or chromosome regions with dis-

Table 2. Attempt of a quantitative characterization of the Prorocentrum triesiinum karyotype

\begin{tabular}{|c|c|c|c|c|c|}
\hline $\begin{array}{c}\text { Chromosome } \\
\text { number }\end{array}$ & $\begin{array}{l}\text { Number of } \\
\text { chromosomes } \\
\text { analyzed }\end{array}$ & $\begin{array}{l}\text { Length }(\mu \mathrm{m}) \\
(\mathrm{m} \pm \mathrm{dt})\end{array}$ & $\begin{array}{c}\text { Relative } \\
\text { length }(\%)\end{array}$ & $\begin{array}{l}\text { Lambda } \\
(\mathrm{m} \pm \mathrm{dt})\end{array}$ & $\begin{array}{l}\text { Coils number } \\
\quad(\mathrm{m} \pm \mathrm{dt})\end{array}$ \\
\hline 1 & 3 & $4.3 \pm(<0.05)$ & 4.8 & $13.5 \pm 0.08$ & $8.5 \pm(<0.1)$ \\
\hline 2 & 3 & $4.4 \pm(<0.05)$ & 4.8 & $10.7 \pm 0.08$ & $8.5 \pm(<0.1)$ \\
\hline 3 & 6 & $4.1 \pm(<0.05)$ & 4.6 & $11.5 \pm 0.13$ & $8.0 \pm(<0.1)$ \\
\hline 4 & 5 & $4.0 \pm(<0.0)$ & 4.5 & $12.3 \pm 0.63$ & $8.0 \pm(<0.1)$ \\
\hline 5 & 2 & $3.9 \pm(<0.05)$ & 4.4 & $12.4 \pm 0.00$ & $8.0 \pm(<0.1)$ \\
\hline 6 & 4 & $3.8 \pm(<0.05)$ & 4.3 & $8.6 \pm 0.04$ & $7.5 \pm(<0.1)$ \\
\hline 7 & 6 & $3.7 \pm(<0.05)$ & 4.1 & $10.5 \pm 0.07$ & $7.5 \pm(<0.1)$ \\
\hline 8 & 5 & $3.5 \pm(<0.05)$ & 3.9 & $9.9 \pm 0.07$ & $7.0 \pm(<0.1)$ \\
\hline 9 & 3 & $3.4 \pm(<0.05)$ & 3.8 & $11.4 \pm 0.08$ & $7.0 \pm(<0.1)$ \\
\hline 10 & 9 & $3.3 \pm(<0.05)$ & 3.7 & $8.2 \pm 0.07$ & $7.0 \pm(<0.1)$ \\
\hline 11 & 5 & $3.2 \pm(<0.05)$ & 3.6 & $8.9 \pm 0.07$ & $7.0 \pm(<0.1)$ \\
\hline 12 & 5 & $3.2 \pm(<0.05)$ & 3.6 & $9.9 \pm 0.07$ & $7.0 \pm(<0.1)$ \\
\hline 13 & 4 & $3.1 \pm(<0.05)$ & 3.5 & $9.7 \pm 0.05$ & $6.5 \pm(<0.1)$ \\
\hline 14 & 12 & $3.0 \pm(<0.05)$ & 3.4 & $9.3 \pm 0.21$ & $6.5 \pm(<0.1)$ \\
\hline 15 & 3 & $3.0 \pm(<0.05)$ & 3.4 & $8.4 \pm 0.05$ & $6.5 \pm(<0.1)$ \\
\hline 16 & 2 & $2.9 \pm(<0.05)$ & 3.3 & $9.2 \pm 0.05$ & $6.5 \pm(<0.1)$ \\
\hline 17 & 8 & $2.9 \pm(<0.05)$ & 3.3 & $8.0 \pm 0.10$ & $6.5 \pm(<0.1)$ \\
\hline 18 & 3 & $2.7 \pm(<0.05)$ & 3.0 & $6.6 \pm 0.08$ & $6.0 \pm(<0.1)$ \\
\hline 19 & 2 & $2.6 \pm(<0.05)$ & 2.9 & $8.3 \pm 0.00$ & $6.0 \pm(<0.0)$ \\
\hline 20 & 3 & $2.6 \pm(<0.05)$ & 2.9 & $7.2 \pm 0.05$ & $6.0 \pm(<0.1)$ \\
\hline 21 & 1 & $2.5 \pm(<0.05)$ & 2.8 & $6.9 \pm 0.00$ & $5.5 \pm(<0.1)$ \\
\hline 22 & 6 & $2.2 \pm(<0.05)$ & 2.5 & $6.1 \pm 0.09$ & $5.0 \pm(<0.1)$ \\
\hline 23 & 10 & $2.1 \pm(<0.05)$ & 2.4 & $5.9 \pm 0.12$ & $5.0 \pm(<0.1)$ \\
\hline 24 & 7 & $2.0 \pm(<0.05)$ & 2.2 & $5.1 \pm 0.12$ & $5.0 \pm(<0.1)$ \\
\hline 25 & 8 & $1.9 \pm(<0.05)$ & 2.1 & $6.1 \pm 0.10$ & $4.5 \pm(<0.1)$ \\
\hline 26 & 4 & $1.9 \pm(<0.05)$ & 2.1 & $5.2 \pm 0.08$ & $4.5 \pm(<0.1)$ \\
\hline 27 & 7 & $1.8 \pm(<0.05)$ & 2.0 & $6.3 \pm 0.12$ & $4.5 \pm .<0.1)$ \\
\hline 28 & 5 & $1.8 \pm(<0.05)$ & 2.0 & $5.5 \pm 0.08$ & $4.5 \pm(<0.1)$ \\
\hline 29 & 11 & $1.7 \pm(<0.05)$ & 1.9 & $4.6 \pm 0.13$ & $4.5 \pm(<0.1)$ \\
\hline 30 & 9 & $1.5 \pm(<0.05)$ & 1.7 & $4.6 \pm 0.09$ & $4.0 \pm(<0.1)$ \\
\hline 31 & 2 & $1.3 \pm(<0.05)$ & 1.4 & $4.1 \pm 0.05$ & $3.5 \pm(<0.1)$ \\
\hline 32 & 1 & $1.0 \pm(<0.05)$ & 1.1 & $2.5 \pm 0.00$ & $3.0 \pm(<0.1)$ \\
\hline
\end{tabular}

tinctive morphological or staining characteristics like in eukaryote sex chromosome. Furthermore, till now no data on sexual mating have been reported in $P$. triestinum.

\section{Abstract}

164 whole mounted "screw like" chromosomes of Prorocentrum triestinum were morphometrically characterized by analyzing their axial length, coil number, diameter, pitch, tilt angle, total wings length and Weibel's lambda coefficient of form. Although the respective 

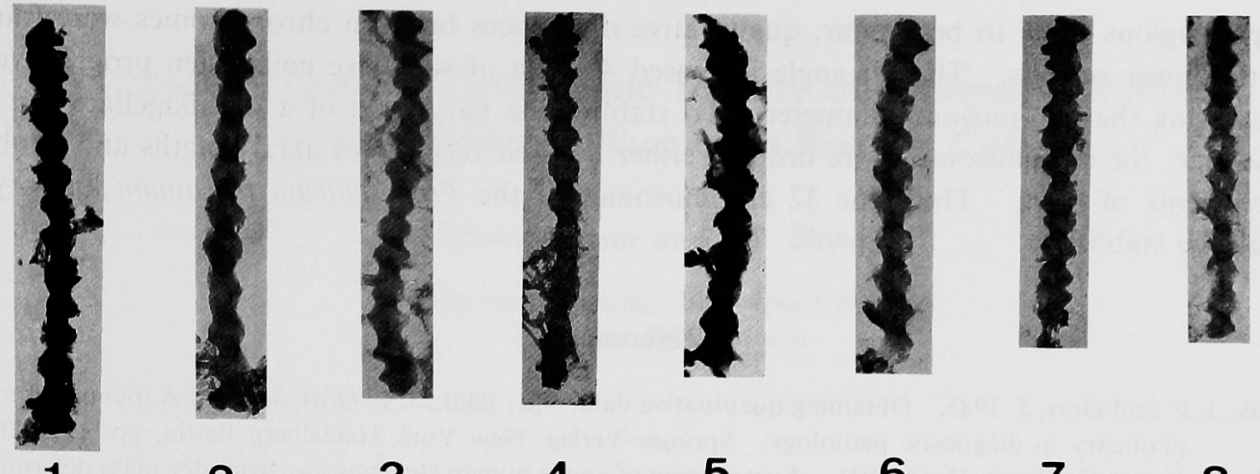

1

2

3

4

5
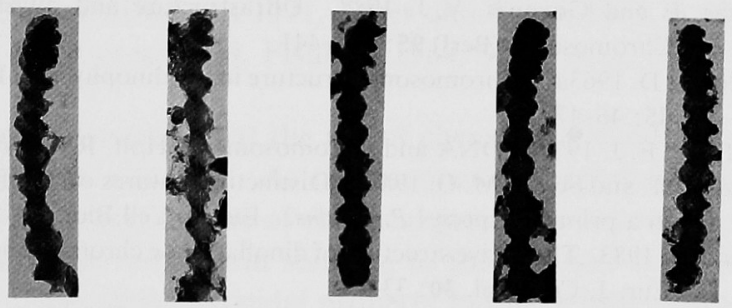

9

10

11

12

13

14

15

16

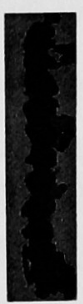

17

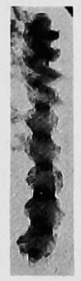

18

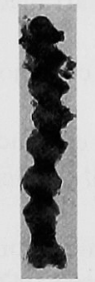

19

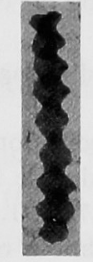

20

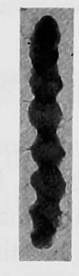

21

22
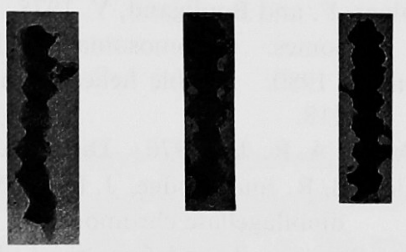

23

24
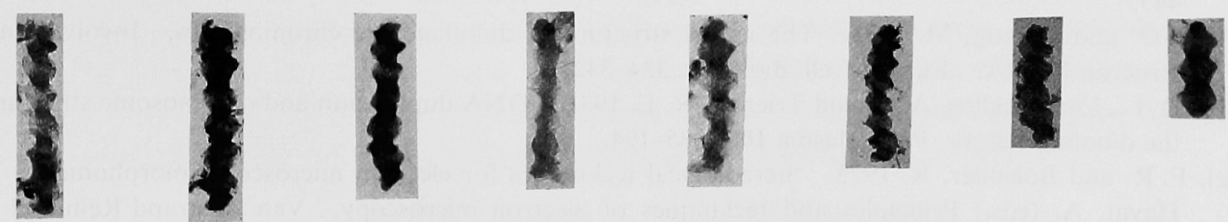

25

26

27

28

29

30

31

32

Fig. 3. Ouantitative karyotype of Prorocentrum triestinum based on the values given in Table 2. 
upper regions show to be similar, quantitative differences between chromosomes were found in the lower regions. The tilt angle increased $4^{\circ}$ each of succesive coils, then progressivelly decreasing the chromosome diameter. To stablish the karyotype of a dinoflagellate for the first time, the chromosomes were ordered either by their respectives axial lengths and lambda coefficients of form. Thus, the 32 chromosomes of the Prorocentrum triestinum karyotype could be stablished.

\section{References}

Baak, J. P. and Oort, J. 1983. Obtaining quantitative data. In: Baak, J. P. Oort, J. (eds.) A manual of morphometry in diagnostic pathology. Springer-Verlag. New York Heidelberg Berlin, pp. 159-180.

Bahr, G. F. and Goulomb, H. M. 1971. Karyotyping of single human chromosome from dry mass determined by electron microscopy. Proc. Nat. Acad. Sci. USA 68: 726-730.

Blank, B. J. 1987. Cell architecture of the dinoflagellate Symbiodinium sp. inhabiting the Hawaiian story coral Montipor verrucosa. Marine Biology 94: 143-155.

Costas, E and Goyanes, V, J. 1987. Ultrastructure and division behaviour of dinoflagellate chromosomes. Chromosoma (Berl) 95 : 435-441.

Dodge, J. D. 1963a. Chromosome structure in the dinophyceae. I. The spiral chromonema. Acrh Mikrobiol. 45: $46-47$.

Du Praw, E. J. 1970. DNA and Chromosomes. Holt, Rinehart and Winston Inc New York. 340 p.

Herzog, M. and Soyer, M. O. 1981. Distinctive features of dinoflagellate chromatin. Absence of nucleosomes in a primitive species $P$. micans. Eur. J. Cell Biol, 23: 295-302.

- and - 1983. The native structure of dinoflagellate chromosomes and their stabilization by $\mathrm{Ca}$ and $\mathrm{Mg}$ cations. Eur. J. Cell Biol. 30 : 33-41.

-, Boletzsky, S. and Soyer, M. O. 1984. Ultrastructural and biochemical nuclear aspects of eukaryote classification: independent evolution of the dinoflagellates as a sister group of the actual eukaryotes ?. Origins Life 13: 205-215.

Lamper, F. Bahr, G. F., Du Praw, E. J. 1969. Ultrastructure of a Burkitt's lymphoma marker chromosome, as investigated by quantitative electron microscopy. Cancer 21: 367-376.

Livolant, F. and Bouligand, Y. 1978. New observations on the twisted arrangement of dinoflagellate chromosomes. Chromosoma 68: 21-44.

— and - 1980. Double helical arrangement of spread dinoflagellate chromosomes. Chromosoma 80: 97118.

Loeblich, A. R. III 1976. Dinoflagellate evolution: speculation and evidence. J. Protozool. 23: 13-28.

Oakley, B. R. and Dodge, J. D. 1979. Evidence for a double helically coiled toroidal chromonema in the dinoflagellate chromosome. Chromosoma 70: 277-291.

Renau-Piqueras, J. and Cervera, J. 1983. Chromatin pattern of isolated human small thymocites. A morphometric and stereologic study. Virchows Arch. (Cell Pathol.) 42: 315-325.

-, Gomez-Perrotta, C., Guerri, C. and Sanchis, R. 1983. Qualitative and quantitative ultrastructural alteration in hepatocytes of rats prenatally exposed to ethanol with special reference to mitochondria, Golgi apparatus and peroxisomes. Virchows Arch. (Cell Pathol.) 405: 237-251.

Rizzo, P. J. 1981. Comparative aspects of basic chromatin proteins in dinoflagellates. Biosystems 14: 433443.

Soyer, M. O. and Herzog, M. 1985. The native structure of dinoflagellate chromosomes. Involvement of structural RNA. Eur. J. Cell Biol. 36: 334-342.

Spector, D. L., Vasconcellos, A. C. and Triemer, R. E. 1981. DNA duplication and chromosome structure in the dinoflagellates. Protoplasma 105: 085-194.

Weibel, F. R. and Bolender, R. 1973. Stereological techniques for electron microscopic morphometry. In: Hayat, A. (eds.) Principles and techniques of electron microscopy. Van Nostrand Reinhold Co., New York, pp. 237-296. 